This document was prepared in conjunction with work accomplished under Contract No. DE-AC09-96SR18500 with the U.S. Department of Energy.

This work was prepared under an agreement with and funded by the U.S. Government. Neither the U. S. Government or its employees, nor any of its contractors, subcontractors or their employees, makes any express or implied: 1 . warranty or assumes any legal liability for the accuracy, completeness, or for the use or results of such use of any information, product, or process disclosed; or 2 . representation that such use or results of such use would not infringe privately owned rights; or 3 . endorsement or recommendation of any specifically identified commercial product, process, or service. Any views and opinions of authors expressed in this work do not necessarily state or reflect those of the United States Government, or its contractors, or subcontractors. 


\title{
SAES ST 909 PILOT SCALE METHANE CRACKING TESTS
}

\author{
J. E. Klein and H. T. Sessions
}

Savannah River National Laboratory: Aiken, SC 29808, james.klein@srnl.doe.gov

Pilot scale (500 gram) SAES St 909 methane cracking tests were conducted to determine material performance for tritium process applications. Tests that ran up to 1400 hours have been performed at $700^{\circ} \mathrm{C}, 202.7 \mathrm{kPa}$ (1520 torr) with a $30 \mathrm{sccm}$ feed of methane, with various impurities, in a 20 vol\% hydrogen, balance helium, stream. A 2.5 vol\% methane feed was reduced below 30 ppm for 631 hours. A feed of 1.1 vol\% methane plus 1.4 vol\% carbon dioxide was reduced below 30 ppm for 513 hours. The amount of carbon dioxide gettered by St 909 can be equated to an equivalent amount of methane gettered to estimate a reduced bed life for methane cracking. The effect of $0.4 \mathrm{vol} \%$ and $2.1 \mathrm{vol} \%$ nitrogen in the feed reduced the time to exceed $30 \mathrm{ppm}$ methane to 362 and 45 hours, respectively, but the nitrogen equivalence to reduced methane gettering capacity was found to be dependent on the nitrogen feed composition. Decreased hydrogen concentrations increased methane getter rates while a drop of $30^{\circ} \mathrm{C}$ in one bed zone increased methane emissions by over a factor of 30. The impact of gettered nitrogen can be somewhat minimized if the nitrogen feed to the bed has been stopped and sufficient time given to recover the methane cracking rate.

\section{INTRODUCTION}

The Savannah River Site (SRS) Tritium Facilities use non-evaporative metal getter beds containing SAES ${ }^{\circledR} \mathrm{St}$ 909 to remove oxygen, carbon monoxide, carbon dioxide, and to crack water, methane, and ammonia contained in various process gas streams. Tritiated methane and ammonia impurities will increase facility emissions unless they are processed before release to the environment. Like the design of the ITER Tokamak Exhaust Processing (TEP) System ${ }^{1}$, SRS process streams will use diffusers/permeators to remove tritium before flow through an impurity processor (a St 909 bed at SRS) followed by further diffuser tritium removal. Methane in a diffuser gas stream may have a deleterious impact on diffuser operation and performance due to the potential coking from methane decomposition.

Previous bench scale St 909 testing, a ten pellet stack per test (6 grams), studied the impact of helium, hydrogen, and nitrogen carrier gases ${ }^{2}$ and of carbon dioxide $^{3}$ on methane cracking. These tests identified important parameters for methane cracking, but are inadequate for predicting performance of full scale beds. Some full scale tests have been completed ${ }^{4}$, but additional tests were needed under different process conditions to determine the long-term ability of St 909 to crack methane prior to additional diffuser processing.

\section{BACKGROUND}

In the absence of diffuser methane decomposition kinetic test data, it is desirable to run the first diffuser(s) under conditions where the gas phase methane decomposition reaction $\left(\mathrm{CQ}_{4}\right.$ going to solid C plus two $\mathrm{Q}_{2}$ where Q is $\mathrm{H}, \mathrm{D}$, or $\mathrm{T}$ ) is not thermodynamically favored. Hydrogen isotope removal by a diffuser can ultimately create conditions favorable for methane decomposition.

Choosing reference states as pure components at 101 $\mathrm{kPa}(1 \mathrm{~atm})$ and $25^{\circ} \mathrm{C}$ and assuming ideal gas conditions, the standard state fugacity equals $\mathrm{P}_{\text {ref }}(101 \mathrm{kPa})$ and component fugacity is equal to its gas phase mole fraction, $\mathrm{y}_{\mathrm{i}}$, times the system pressure, $\mathrm{P}$. Carbon activity is taken as unity since its variation with pressure dependence is assumed to be small. The methane decomposition equilibrium constant, $\mathrm{K}_{\mathrm{a}}$, is first order in pressure, second order in hydrogen isotope mole fraction and inversely proportional to methane mole fraction.

Figure 1 shows equilibrium conditions for methane decomposition at $101 \mathrm{kPa}$ were $\mathrm{K}_{\mathrm{a}}$ values were calculated for protium using tabulated values for heat capacity polynomial coefficients and heat and free energies of formation, at $25^{\circ} \mathrm{C}$ and $101 \mathrm{kPa} .^{5,6}$ Figure 1 gas compositions to the left of an isotherm favor methane decomposition while compositions to the right favor methane reformation.

For various feed gas scenarios, mass balance lines, where protium was removed from the mixture, were plotted on Figure 1. The intersection of the mass balance line and the assumed $500^{\circ} \mathrm{C}$ diffuser "hot spot" isotherm indicated the minimum protium concentration needed to suppress the methane decomposition reaction. For these tests, the first diffuser exit composition (the feed to the St 
909 bed) was rounded to 20 vol\% protium to keep the diffuser from operating in the coking regime.

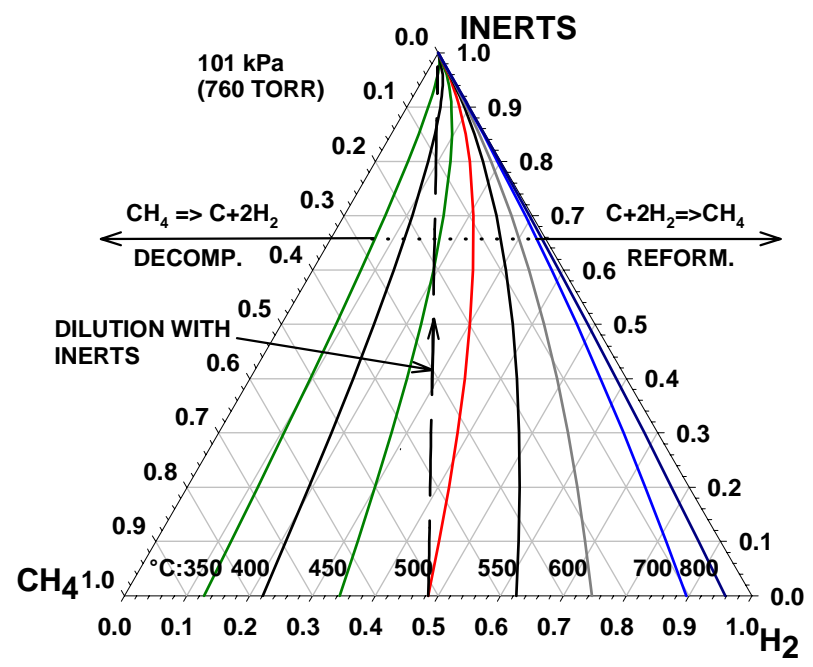

Figure 1. Methane Decomposition Equilibrium Plot

The purpose of these cold (non-radioactive) tests was to determine if St 909 could reduce methane concentrations in $20 \mathrm{vol} \%$ protium below $30 \mathrm{ppm}$ and determine the impact of impurities on methane cracking.

\section{EXPERIMENTAL}

Pilot scale (500 grams St 909) test beds were fabricated from 150 cc 316L stainless steel sample cylinders. Thermowells for type K thermocouples (TCs) were made from small pieces of tubing and attached to the bed exterior near the bottom, middle, and top of a bed while another TC was inserted into the top of the bed and in contact with the top bed pellets. A bed was installed in a 3-zone, vertical furnace for testing. The test system has been described previously ${ }^{2,3}$ with an Agilent Technologies 3000A Micro GC added for methane gas analyses. Gas standards of nominally $30 \mathrm{ppm}, 20,000 \mathrm{ppm}$, and 25,000 ppm methane were used for GC calibration.

A bed was activated by flowing $30 \mathrm{sccm}$ argon through the bed at ambient temperature to displace air followed by a nominal $2.5^{\circ} \mathrm{C}$ per minute temperature ramp to $700^{\circ} \mathrm{C}$. The bed was at temperature for nominally 12 hours before the start of a test.

Before a test, the bed was isolated with an argon fill at $700^{\circ} \mathrm{C}$ and a pressure of $202.7 \mathrm{kPa}$ (1520 torr). After a steady feed gas mixture composition at $30 \mathrm{sccm}$ was obtained, it was routed through the test bed. Periodically during testing, the bed was bypassed to verify feed composition stability. GC standards were periodically run during testing to verify instrument calibration.
Feed gas mixtures contained various amounts of methane, carbon dioxide, and nitrogen impurities along with $20 \mathrm{vol} \%$ protium and a balance of helium. Test 1 had 2.5 vol\% methane and Test 2 had 1.1 vol\% methane plus 1.4 vol\% carbon dioxide. Test 3 , part A (Test 3A) had $1.1 \mathrm{vol} \%$ methane plus $2.1 \mathrm{vol} \%$ nitrogen while for Test 3B, the $0.63 \mathrm{sccm}$ nitrogen flow was stopped and then restarted for Test 3C. Test 4A had 1.1 vol\% methane plus $0.4 \mathrm{vol} \%$ nitrogen while Test $4 \mathrm{~B}$ had the $0.12 \mathrm{sccm}$ nitrogen flow stopped. Test $4 \mathrm{C}$ added $200 \mathrm{ppm}$ water to the Test $4 \mathrm{~B}$ feed while Test $4 \mathrm{D}$ added $1.1 \%$ carbon dioxide to the Test $4 \mathrm{C}$ feed. Test $4 \mathrm{E}$ continued Test 4D conditions with greatly reduced hydrogen flow due to a mass flow controller problem.

\section{RESULTS}

As activation bed temperatures approached 615 to $625^{\circ} \mathrm{C}$, temperature increases exceeding the furnace ramp rate were observed. The internal TC first indicated this temperature increase while the middle TC registered a maximum temperature of $715^{\circ} \mathrm{C}$ : an increase of almost $100^{\circ} \mathrm{C}$ in four minutes. This exothermic reaction has been seen before for St 909 and was anticipated.,

Water, carbon dioxide, carbon monoxide/nitrogen, and hydrogen were released where desorbed/released during activation, but most surprisingly was methane releases. Figure 2 shows methane releases as a function of bed middle temperature: the data between 680 and $720^{\circ} \mathrm{C}$ appear as a discontinuous function due to the rise and fall in temperature due to the exothermic reaction. The Test 1, 2, and 4 St 909 beds released approximately $8.3 \mathrm{scc}$ methane while the Test 3 bed released only $0.2 \mathrm{scc}$ methane. The Test 1, 2, and 4 St 909 was from the same production lot while the Test 3 St 909 was from a different material lot.

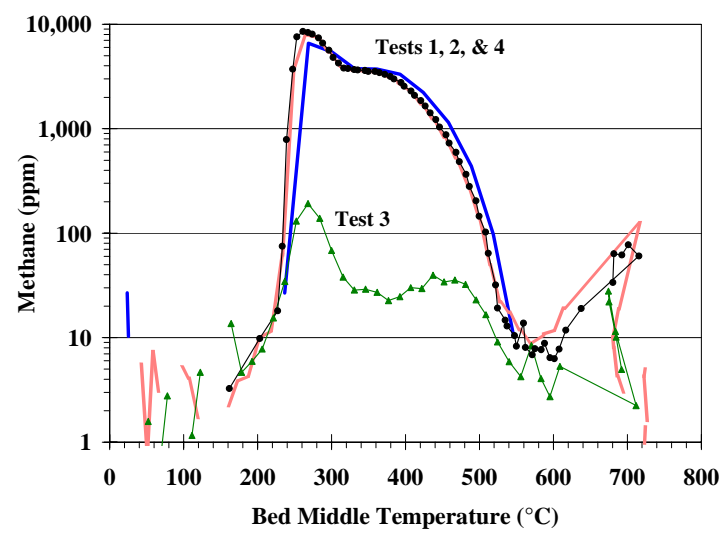

Fig. 2. Bed Activation Methane Releases 
Figure 3 shows the outlet GC methane concentration versus time for Tests $1,2,3 \mathrm{~A}$, and $3 \mathrm{~B}$ and Table I summarizes some of the results when the bed exit concentration reached $30 \mathrm{ppm}$. The methane impurity equivalence (MIE) parameter was derived to relate the amount of carbon dioxide or nitrogen gettered to an equivalent amount of methane gettered: the difference in the quantity of methane gettered in Test 1and another test, divided by the quantity of the other impurity gettered.

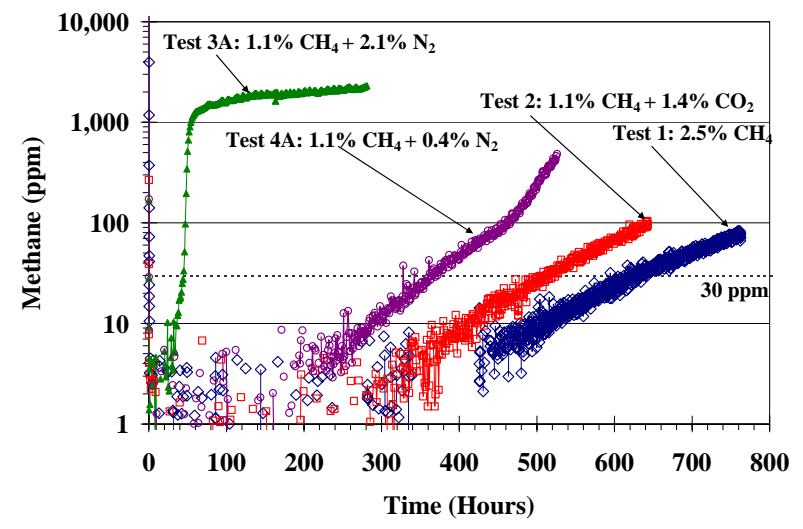

Fig. 3. Test Results Versus Time

Table I. Results at/for 30 ppm Methane Outlet

\begin{tabular}{|l|c|c|c|c|}
\hline \multicolumn{1}{|c|}{ Test \# } & 1 & 2 & $3 \mathrm{~A}$ & $4 \mathrm{~A}$ \\
\hline Time (hours) $^{2}$ & 631 & 513 & 45 & 362 \\
\hline $\mathrm{CH}_{4}$ Gettered $^{\mathrm{a}}$ & 55.7 & 16.2 & 1.78 & 14.0 \\
\hline $\mathrm{CO}_{2}$ Gettered $^{\mathrm{a}}$ & n/a & 24.9 & n/a & n/a \\
\hline $\mathrm{N}_{2}$ Gettered $^{\mathrm{a}}$ & n/a & n/a & 3.46 & 5.12 \\
\hline $\mathrm{MIE}$ & n/a & $1.59^{\mathrm{b}}$ & $15.6^{\mathrm{c}}$ & $8.15^{\mathrm{c}}$ \\
\hline
\end{tabular}

${ }^{\text {a }}$ STP-L/kg-St 909

${ }^{\mathrm{b}} \Delta\left(\mathrm{STP}-\mathrm{L} \mathrm{CH}_{4}\right)$ per STP-L $\mathrm{CO}_{2}$

${ }^{\mathrm{c}} \Delta\left(\mathrm{STP}-\mathrm{L} \mathrm{CH}_{4}\right)$ per STP-L $\mathrm{N}_{2}$

Figure 4 shows Figure 3 results replotted using the MIE factors from Table I versus equivalent methane cracked. Figures 5 and 6 show the complete results for Test 3 and Test 4, respectively, which include transient losses of hydrogen flow, pressure/flow control, furnace power, or the loss of one furnace zone.

Figure 5 shows that when the nitrogen flow was stopped at the end Test 3A (282 hours), the methane outlet concentration decreased and further decreased when the hydrogen flow was lost at 594 hours. During the time when system hydrogen flow and back-pressure were unstable (pressure oscillations between 618 to 670 hours), the methane concentration increased, but then decreased when control was re-established. At 971 hours and 36.89 STP-L methane cracked, the methane concentration again exceeded 30 ppm. Between 1128 and 1149 hours, an open TC circuit shut off the furnace.

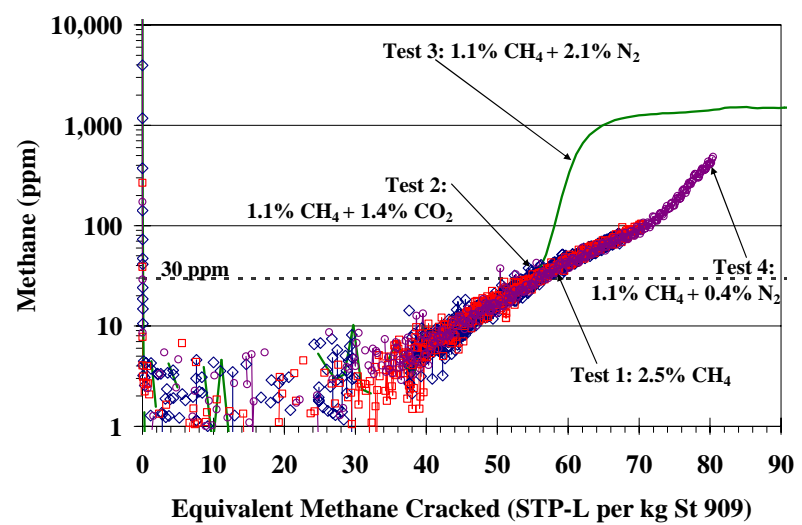

Fig. 4. Test Results Versus Equivalent Methane Cracked

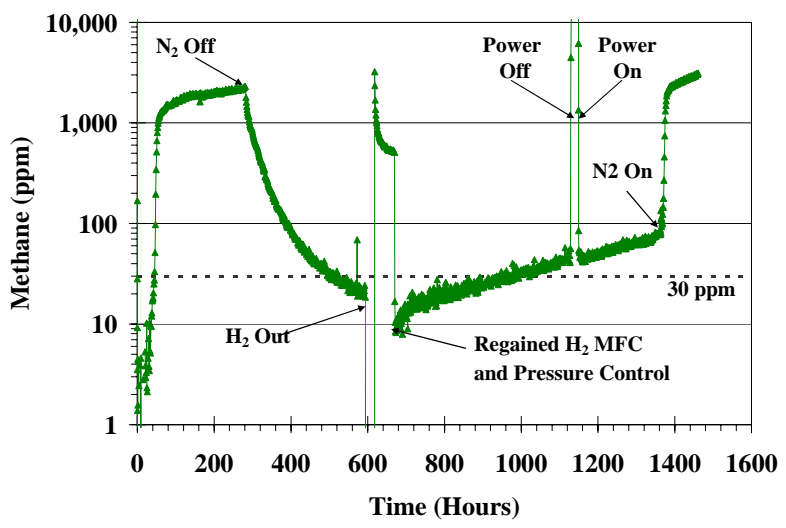

Fig. 5. Complete Test 3 Results

Figure 6 shows that when the nitrogen flow was stopped at the end Test 4A (526 hours), the methane outlet concentration decreases and decreased below 30 ppm at 716 hours. A blown fuse for the bottom furnace zone between 867 and 989 hours showed increased methane concentration which dropped below 30 ppm once the fuse was replaced. The addition of $198 \mathrm{ppm}$ water to the feed stream at 1010 hours had no discernable impact on gettering performance. The impact of adding 1.1 vol\% carbon dioxide at 1201 hours was somewhat obfuscated by the loss of building power between 1217 and 1300 hours, but the rate of increase in outlet methane concentration was greater than before the introduction of the carbon dioxide. At 1061 hours and 39.51 STP-L methane cracked, the methane concentration again 
exceeded $30 \mathrm{ppm}$. The hydrogen flow was stopped at 1393 hours which produced a rapid decrease in methane outlet concentration.

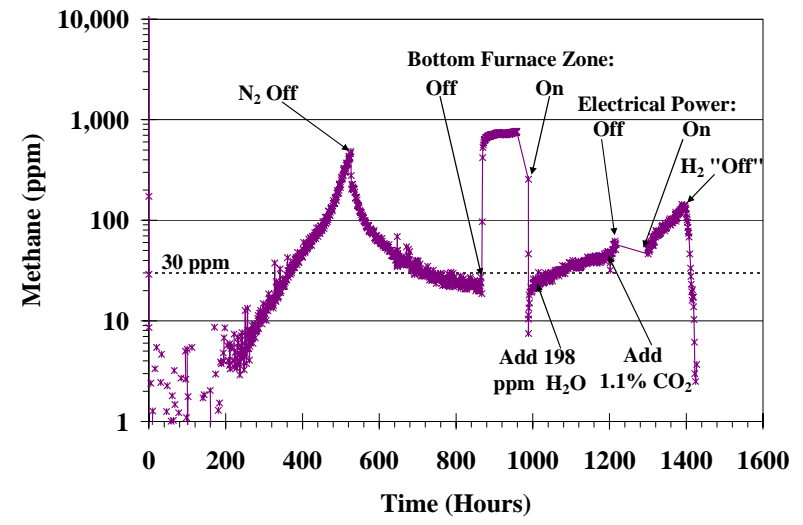

Fig. 6. Complete Test 4 Results

\section{DISCUSSIONS}

These tests showed that a fresh St 909 bed is capable of reducing methane concentrations to less than $30 \mathrm{ppm}$ in a $20 \mathrm{vol} \%$ hydrogen stream. Test 2 showed that with the same carbon feed rate as Test 1, 30 ppm breakthrough was reached earlier than in Test 1 due to the oxygen in the carbon dioxide. The MIE of carbon dioxide being 1.59 instead of 3.00 was interpreted as oxygen being gettered faster than carbon by the St 909 .

Test 3 and Test 4 showed nitrogen had a greater impact on methane cracking than carbon dioxide and the nitrogen MIE was concentration dependent. The effect of nitrogen on methane cracking can be mitigated by stopping the nitrogen supply to the bed and allowing the nitrogen to presumably diffuse from the surface into the bulk allowing methane outlet concentrations to be again below $30 \mathrm{ppm}$.

Figure 7 contains the Figure 4 Test 1 data along with Test 3 data shifted by 18.80 STP-L methane cracked (55.68 from Test 1less 36.89 from Test 3B) illustrates the minimal impact the previously gettered nitrogen has on methane cracking once the Test 3B data drop below 30 ppm.

Figure 7 also shows the Test 4 data shifted by 16.17 STP-L methane cracked (55.68 from Test 1less 39.51 from Test 3B) along with the Test 4D incorporating the carbon dioxide MIE value from Table I illustrating again the minimal impact the previously gettered nitrogen has on methane cracking once the Test $3 \mathrm{C}$ data drop below 30 $\mathrm{ppm}$. There was no significant impact on methane cracking by the 0.22 STP-L of water cracked by the St 909.

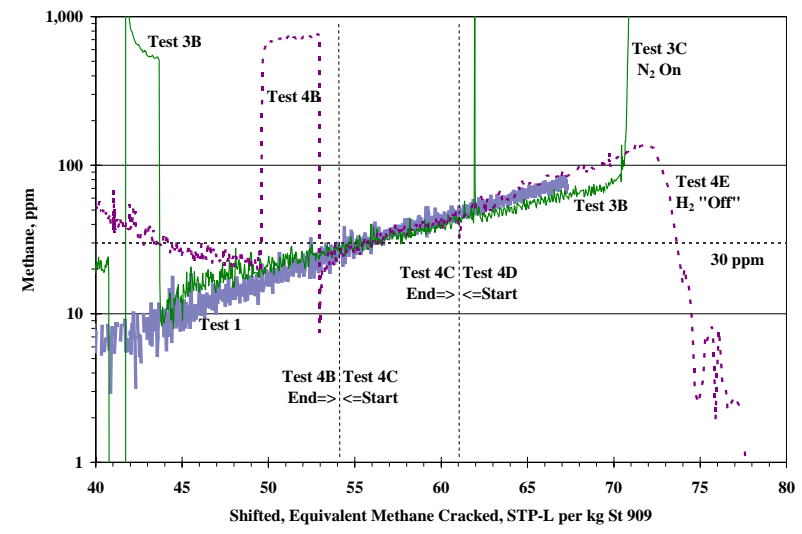

Fig. 7. Methane Cracking on Partially Consumed St 909

The $30^{\circ} \mathrm{C}$ temperature drop at the bed bottom when its heater zone was lost during Test $4 \mathrm{~B}$ illustrates the sensitivity of methane cracking on temperature. Temperature gradients within larger scale St 909 beds must be anticipated since many production processes utilize single-zone vessel furnaces. The pilot scale results mostly like illustrate "best achievable" since larger beds will likely have regions at lower temperatures if controlled to the same $700^{\circ} \mathrm{C}$ temperature.

The start of Test 3C was interesting in that once the nitrogen flow was restarted, the methane outlet concentration increased to the level where Test $3 \mathrm{~A}$ ended, nominally $2300 \mathrm{ppm}$, in the relatively short time of 20 hours. The difference between the end of Test $3 \mathrm{~A}$ and the beginning of Test $3 \mathrm{C}$ is the larger rate of increase of outlet methane concentration for Test $3 \mathrm{C}$ than for Test $3 \mathrm{~A}$, presumably due to the gettered carbon and additional nitrogen inhibiting methane cracking.

Hydrogen suppresses the methane decomposition reaction and the reduction of hydrogen fed to the St 909 bed reduced the methane outlet concentration as illustrated in Figures 5 and 6.

\section{CONCLUSIONS}

St 909 can reduce methane feed concentrations to below $30 \mathrm{ppm}$ at $700^{\circ} \mathrm{C}$, with 20 vol\% hydrogen, under low flow conditions, to remove impurities and effectively reduce tritium emissions. The reduction in bed methane cracking capacity can be estimated if the amount carbon dioxide in the feed gas is known utilizing the define MIE 
ratio. Nitrogen can have a significant, negative impact on St 909 methane cracking efficiency and its impact is dependent on the nitrogen concentration in the feed gas. The impact of nitrogen gettered during St 909 methane cracking can be somewhat reversed if the nitrogen feed to the bed is stopped long enough to recover previous methane cracking performance.

\section{ACKNOWLEDGMENTS}

The authors would like to thank Jody Dye and Joel Jones for there support of this work. This paper was prepared in connection with work done under Contract No. DE-AC09-96SR18500 with the U. S. Department of Energy.

\section{REFERENCES}

[1] "Design Description Document (DDD): Tritium Plant and Detritiation Systems WBS 32A

Tokamak Exhaust Processing,” ITER Project Document N 32 DDD 32 01-06-29 R0.1.

[2] J. E. Klein, "SAES St909 Bench Scale Methane Cracking Tests," Fusion Sci. \& Technol., 41, 998 (2002).

[3] J. E. Klein, "Bench Scale SAES St 909 Tests for Methane and Carbon Dioxide Removal From Helium, Hydrogen, and Nitrogen Streams," AIChE Spring Meeting, Orlando, FL (2006).

[4] R. H. Hsu and J. S. Holder, "Testing of a Prototype SAES St 909 Getter Bed for Conditioning Gas to a Tritium Stripper System,” Fusion Technol., 48, 171 (2005).

[5] E R. C. Reid, J. M. Prausnitz, and B. E. Poling. "The Properties of Gases and Liquids" $4^{\text {th }}$ Ed. Appendix A, McGraw-Hill, New York, U.S.A. (1987)

[6] "CRC Handbook of Chemistry and Physics, 55 Ed.”, Thermodynamic Properties of the Elements and Oxides, p. D-56, CRC Press, Cleveland, OH (1975).

[7] E. J. Larson, K. J. Cook, J. R. Wermer, and D. G. Tuggle, "Nitriding Reactions with a Zr-Mn-Fe Metal Getter," J. Alloys and Compounds, 330332, 897 (2002). 